\title{
VI.
}

Aus dem pharmakologischen Institut zu Heidelberg.

\section{Vergleichende Untersuchungen über die kumulative Wirkung der Digitaliskörper.}

\author{
Von \\ Dr. Albert Fraenkel (Badenweiler). \\ (Mit 6 Abbildungen im Text.)
}

Der Tierversuch hat den Mechanismus klargelegt, duroh den Digitalis die Stauungserscheinungen in so vielen Fällen von Kreis. laufsstörung zu beseitigen vermag. Dabei konnte sich die experimentelle Analyse der Digitaliswirkung nur auf das Studium der akuten Vergiftung stïtzen; denn nur wenn alle Veränderungen, in kurzen Zeitraum zusammengedrängt, sich rasch bis zu einer Höhe entwickeln, in der sie den messenden Methoden der Physiologie zugänglich sind, war es natülich möglich, die einzelnen Wirkungen auf die verschiedenen Faktoren des Kreislaufs auseivander zu halten, die Beziehungen der gesteigerten Herzarbeit und der gleichzeitigen Gefäßwirkungen zur Blutdrucksteigerung festzustellen und die Bedeutung der begleitenden Veränderungen der Pulsfrequenz aufzuklären. Doch bedarf der akute Vergiftungsversuch einer Ergänzung durch das Studium der Veränderungen, welche kleine und nicht akut vergiftende Gaben an gesunden und kranken Tieren hervorrufen. Denn über den Verlauf der ersten Kreislaufssymptome sowie taber die Mögliohkeit, den therapeutiseh verwertbaren ersten Grad der Wirkung längere Zeit hindurch festzuhalten, gibt nicht die Einverleibung einmaliger großer, sondern die wiederholte Anwendung kleiner Gaben Aufsehluß.

Von diesen klinisch wichtigen Gesichtspunkteu aus, wurden die folgenden Versuche unternommen. Vor allem war es dabei die Auf. gabe, Eintritt und Verlauf der ersten Erscheinungen am Zirkulationsapparat zu beobachten und diese mit dem Allgemeinbefinden der Versuchstiere und insbesondere mit dem zeitlichen Auftreten von toxischen Nebenwirkungen zusammenzuhalten.

Als die ersten Symptome der Digitaliswirkung am Kreislaufe kennen wir die Blutdrucksteigerung und die sie begleiteude Pulsver- 
langsamung. Von diesen beiden Kardinalsymptomen ist aber nur das eine, die Veränderung der Pulsfrequenz, ohne Schädigung der Versuchs. tiere einer länger dauernden Beobachtung zugänglicb. Die Steigerung der Herzarbeit, die als das therapeutisch entscheidende Moment das größte Interesse beanspruchen würde, kann am intakten Tier nicht gemessen werden und ebenso stellen sich dem Versuche, etwa in wochenlanger Beobachtung die Blutdrucksteigerung verfolgen zu wollen, unüberwindliche Schwierigkeiten entgegen. Hingegen kann die Beobachtung der Pulsfrequenz im gewissem Sinne die Beobachtung der anderen Kreislaufsfaktoren ersetzen, da sie einen sicberen Gradmesser für die Veränderung der Kreislaufsverhältnisse durch Digitaliskörper abgibt. Wir wissen, daß das isolierte Warmbltiterherz im Gegensatz zum Froschberzen durch die Digitaliskörper nicht direkt verlangsamt wird 1). Die Pulsverlangsamung in den ersten Stadien der Digitaliswirkung am Warmblüter ist also nur durch den Erregungszustand des Vaguszentrums bedingt und dieser wird bekanntlich von der Blutdruckhöbe beherrscht. Wir sehen deshalb in der Pulsverlangeine Regulationseinrichtung, welche den blutdrucksteigernden Einflüssen im Beginn und auf der Höbe der Digitaliswirkung entgegenarbeitet. Erst wenn die Grenzen der therapeutisch verwertbaren Wirkung uberschritten sind, versagt diese Gegenwirkung, das Herz folgt dann dem hemmenden Zligel des Vagus nicht mehr und der Puls wird äuBerst frequent. Wir haben daher das Recht, aus dem Eintritt und aus der Stärke der Pulsverlangsamung auf das Besteben einer Digitaliswirkung und auf den Grad derselben zu schließen.

In den folgenden Versuchen, die an gesunden Katzen ausgeführt wurden, ist somit das Verhalten der Pulsfrequenz als Gradmesser der Kreislaufswirkung benutzt. Gleichzeitig mit der Pulsverlangsamung war bei allen Versuchstieren auch eine zweifellose Verstärkung des Herzspitzenstoßes gegenüber der Norm zu fühlen; im therapeutisch in Betracht kommenden ersten Stadium ist diese Wirkung nach kleinen Gaben der Digitaliskörper eine sehr eklatante, aber einer einwandsfreien quantitativen Messung ist diese Veränderung nicht zugänglich.

Auch die Pulsfrequenz kann an den Versuchstieren durch einfache Palpation nicht mit genügender Sicherheit festgestellt werden. Schon im normalen Zustand ist die Frequenz der Herzschläge von Kaninchen, Katzen und selbst Hunden eine so hohe, daß eine genaue Zählung mit der Hand schwierig ist; wenn der Puls in der

1) Vergl die vorangehende Mitteilung von R. Gottlieb und R. Magnus. 
Digitaliswirkung unregelmäßig wird, ist die Zählung erst recht unmöglich. Speziell ftir Katzen, die ich als Versuchstiere benutzte, habe ich mich durch einige Versuchsreihen therzeugen können, daß bei der normal hohen Pulsfrequenz der Tiere -200 bis 250 Pulse in der Minute - durch den tastenden Finger stets nur ungenaue und meist zu niedrige Frequenzen ermittelt werden. Angaben, die auf einfacher Zählung durch Palpation beruhen, verdienen daher nicht viel Vertrauen. Nach einigen Vorversuchen bediente ich mich deshalb zur Pulszåhlung aussohließlich des Kardiographen. Es gelingt nach einiger Übung bei Katzen, die an das Laboratorium bereits gewöhnt sind, ganz leicht, die Tiere mit der linken Hand in bequemer Seitenlage so festzuhalten, daß der mit der rechten Hand auf die Gegend des deutlichen Herzspitzenstoßes aufgesetzte Kardiograph die Pulse deutlich auf dem Kymographion verzeichnet. Registriert man gleichzeitig mit einem Zeitsohreiber die Sekunden, so können die Herzschlăge während mehrerer Perioden einer kardiographischen Kurve genau ausgezählt werden. Das Tier kommt dabei nicht in eine unbequeme Lage und sträubt sich nicht, so daß die Pulsfrequenz nicht etwa wie beim Aufbinden durch die Erregung des Tieres verändert wird. Es gelingt so in der Tat, verläßliche Normalwerte zu bekommen. Einzelne widerspenstige Tiere mtissen ausgeschaltet werden.

Mit der beschriebenen Methode sind die Pulszahlen in den wochenlangen Versuchen ermittelt, die ich mit Digitoxin Merck, Digitalinum verum von Böhringer \& Söhne, Strophanthin Merok und Strophanthin Böhringer anstellte. Die Substanzen wurden subkutan injiziert; die Giftlösungen kamen dabei in solcher Verdtunnung zur Anwendung, daß auch das Digitoxin die Versuche nicht durch lokale Reizwirkung stören konnte.

Verfolgt man nach einer wirksamen Dosis dieser zur Gruppe des Digitalins gehörigen Substanzen das Verhalten der Pulsfrequenz durch längere Zeit, so fällt vor allem die Dauer der Nachwirkung auf. Ist die Pulsverlangsamung und Verstärkung: der Herztätigkeit einmal eingetreten, so wird sie tagelang festgehalten,',vorausgesetzt, daß die Dosis nicht eine derart toxische war, daß sich an die Pulsverlangsamung alsbald schwere Vergiftungserscheinungen anschließen. Werden ferner kleine Gaben, die als Einzeldosen noch durchaus ohne Wirkung auf die Pulsfrequenz bleiben, täglich fortgegeben, so tritt die Pulsverlangsamung nach einer je nach der Gabengröße wechselnden Zeit ein und nimmt allmählich zu. Der Digitaliskörper wird also durch „Kumulierung" wirksam. Es war nun für jedes einzelne Präparat zu untersuchen, inwieweit es möglich ist, eine 
solche Kreislaufswirkung am gesunden Tier bei tãglich gleichbleibenden Gaben innerhalb der therapeutisch verwertbaren Grenzen festzuhalten, oder nach welcher Zeit bei der betreffenden Dosis allmählich Vergiftung entsteht. Weiter war darauf zu achten, ob nicht andererseits die anfangs wirksamen Gaben allmählich unwirksam werden, also Gewöhnung an das Mittel eintritt. Die Beobachtungen zeigten, daß selbst in monatelangen Versuchen nichts zu sehen war, was auf eine Angewöhnung hindeutete, daß sich aber bei allen untersuchten Substanzen eine Dosis ermitteln ließ, bei der die Kreislaufswirkung wochenlang ohne Nebenerscheinungen anhielt und die Tiere sonst normal blieben, ein Resultat, das den Erfahrungen mit fortgesetzter Anwendung kleiner Digitalisgaben am Menschen entspricht. Gingen wir hingegen in unseren Versuchen nur um ein weniges tuber die geeignete tägliche Dosis hinaus, so blieben nur die ersten Gaben noch innerhalb der Grenzen therapeutischen Effektes; bei fortgesetzter Darreichung verlangsamt sich der Puls mehr und mehr, wird unregelmäBig und endlich treten im Ansehluß an eine Injektion die ersten Erscheinungen der Vergiftung, Salivation und Erbrechen ein. Die Tiere verweigern die Nahrung und werden krank. Die kumulative Wirkung ist zur Geltung gekommen.

Wir kennen diese ärztlicherseits geftirchteten Erscheinungen vom Menschen her. Dennoch glauben wir, dal das experimentelle Studium der kumulativen Digitaliswirkung an gesunden Tieren genug: des Interesses bietet. Von den zufälligen Beobachtungen am Mensohen hat der planmäßig angelegte Tierversuch vor allem voraus, daß er das Gesetzmäßige in den Erseheinungen der Kumulierung hervortreten läßt. An dem gleichmäßigen Tiermaterial fallen die Verschiedenheiten der individuellen Disposition fort, dann aber können die Kreislaufsveränderungen an gesunden Tieren nur auf die angewandte Substanz bezogen werden, während man es bei der Anwendung der Digitalis in pathologischen Fällen mit ungleich komplizierteren Bedingungen zu tun hat. Das Studium der kumulativen Wirkung unter möglichst einfachen und planmäßig abgestuften Bedingungen der Darreichung kann deshalb als Grundlage zur Beurteilung der verwickelteren Verhältnisse am Menschen dienen.

Weiter ergibt sich die Aufgabe, die einzelnen reinen Digitalis. körper in bezug auf die Zeit des Eintritts und die Dauer ihrer Wirkung sowie in bezug auf ihre kumulativen Eigenschaften untereinander zu vergleichen. Aus einem derartigen Vergleiche an einem gleichmäßigen Beobachtungsmaterial können sich spezielle Indikationen ftur die Anwendung der einzelnen reinen. Körper ergeben. Von be- 
sonderem Interesse ist es ferner, schwerlösliche Substanzen wie das Digitoxin, mit den leicht wasserlöslichen Strophanthinen in Bezug: auf die Schnelligkeit und Dauer der Wirkung zu vergleichen, um uiber den Anteil der Resorptions- und Ausscheidungsgeschwindigkeit an den Erscheinungen ein Urteil zu gewinnen.

Es liegen bisher nur wenige und gelegentliche Untersuchungen in der angedeuteten Richtung vor. Nur eine Arbeit von v. d. Heide ${ }^{1}$ ) unter der Leitung von Stokvis ist von ähnlichen Gesichtspunkten ausgegangen. Der Wert dieser älteren Untersuchung wird aber dadurch beeinträchtigt, daß nur ein unreines "Digitalin" neben Helleborein zur Anwendung kam. Die Versuche v. d. Heides mit diesem Digitalinextrakt können wir aber auch deshalb nicht zum Vergleich mit unseren Versuchen heranziehen, weil vielfach am gleichen Tiere mit der Darreichung per os und der subkutanen Injektion abgewechselt wurde, die Wirkung bei den beiden Wegen der Zufuhr aber eine durchaus verschiedene ist. Die Helleboreinversuche stimmen im wesentlichen mit den von uns gewonnenen Ergebniasen tuberein; nur von der Angewöhnung, die v. d. Heide konstatiert haben will, konnten wir in unseren ungleich längeren Versuchsreihen mit den genannten Substanzen nichts bemerken.

\section{Versuche mit Digitoxin.}

Das in Wasser so gut wie unlösliche Digitoxin. puriss. cryst. Merek wurde in Alkohol gelöst und die alkoholische Stammlösung für die einzelnen Versuchsreihen in entsprechender Weise mit Wasser verdünnt.

Bei Digitoxin tritt der kumulative Charakter der Digitaliswirkung wohl am dentlichsten hervor. Nicht bloß bei der Darreichung per os, auch nach subkutaner Injektion dauert es immer einige Zeit ehe die Gabe ihre Wirkung entfaltet. Das gilt auch für Dosen, die schon Nebenerscheinungen hervorrufen; auch da stellt sich das Erbrechen, das erste und regelmäßige Zeichen dafür, daß eine toxische Gabe im Blute zirkuliert, niemals vor Ablauf von etwa einer Stunde ein. $\mathrm{Zu}$ den giftempfindlichen Apparaten des Herzens dringt aber das Digitoxin offenbar noch weit langsamer vor, denn die fuhlbare Verstärkung der Herztätigkeit und die meßbare Pulsverlangsamung treten nach "therapeutischen" und selbst nach bereits toxischen Gaben nicht vor 24 Stunden auf. Nur nach mehrfach letalen Dosen wird die Herzwirkung rasoher manifest und zwar desto schneller, je mehr Digitoxin auf einmal in die Zirkulation gerät; Gaben von z. B. $0,5 \mathrm{mg}$ pro $\mathrm{kg}$ Katze töten schon nach

1) Dieses Archiv. Bd. XIX. S. 127. 1885. 
Untersuchungen über die kumulative Wirkung der Digitaliskörper. $\quad 89$

6-12 Stunden unter enormer Pulsverlangsamung ${ }^{1}$ ). Ich führe zur Illustration des verzögerten Eintritts der Kreislaufswirkung nach einer einmaligen etwa minimal-letalen Digitoxindosis von $0,08 \mathrm{mg}$ pro $\mathrm{kg}$ das beistehende Diagramm vor und füge demselben einige Stichproben aus den kardiographisehen Kurven bei, aus denen die Pulsfrequenz ausgezählt wurde.

Diagramm Versuch 18 (4. Febr. 1903): Wirkung einer Einzeldosis Digitoxin $0,08 \mathrm{mg}$ pro $\mathrm{kg}$. Pulsverlangsamung am 3. Tage nach der Injektion, Tod am 10. Tage.

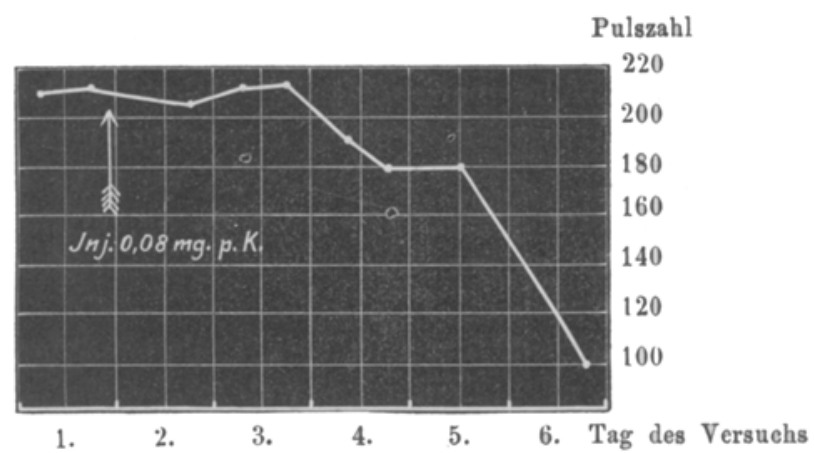

Fig. 1.

Kardiographische Kurven zu Versuch 18.

Normal $=216$ Pulse pro Min.

Vor der Injektion.

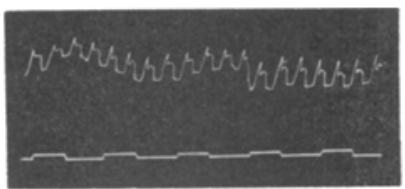

210 Pulse pro Min.

2. Tag nach der Injektion.

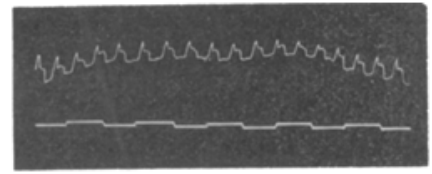

156 Pulse pro Min.

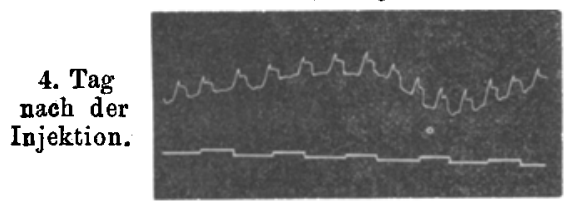

Fig. 2.
162 Pulse pro Min.l

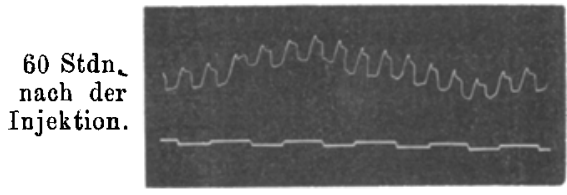

96 Pulse pro Min.

5. Tag nach der Injektion.

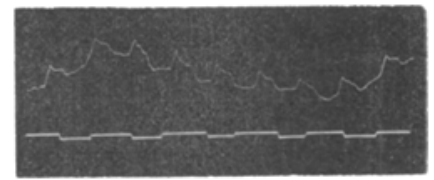

1) Vergl. Koppe, dieses Archiv. Bd. XII. S. 2 i4. 
Wie den verzögerten Eintritt der Pulsverlangsamung, zeigt der angeführte Versuch auch die zweite charakteristische Eigentlumlichkeit der Digitoxinvergiftung, die lange Nachwirkung einer einmaligen toxischen Gabe. Es danerte 60 Stunden, bis die Pulswirkung nach der subkutanen Injektion eintrat, dann aber blieb dieselbe 7 Tage lang bestehen bis zum Tode des Tieres, das unter kolossaler Pulsverlangsamung einging. Ist also das Digitoxin einmal in toxisoher Konzentration ins Blut gelangt, so wird zwar die Wirkung nur langsam manifest, aber sie wird dann auch lange festgehalten, um entweder bei letalem Ausgang sich bis zum Tode zu vertiefen oder auch vor dem Ende ungemein frequenten und irregulären Pulsen Platz zu machen. Erfolgt nach einer einmaligen wirksamen Dosis Erholung, so kehrt die Pulsfrequenz dementsprechend auch nur langsam zur Norm zurück. Das Digitoxin, wenn es einmal ins Herz aufgenommen ist und daselbst seine Wirkung entfaltet, wird offenbar nur sehr langsam wieder vom Herzen abgegeben oder anderweitig unsehädlich gemacht.

Für die Intensität der sich entwickelnden Symptome kommt es nun keineswegs allein darauf an, welche Menge des Giftes während der betreffenden Zeit im Blute kreist, sondern der Effekt hängt auch sehr wesentlich von der zeitlichen Verteilung der Dosis ab. Obgleich also die Wirkung weder in dem einen noch im anderen Falle sogleich manifest wird, kann die gleiche Menge, auf einmal gegeben, nach einigen Tagen zum Tode führen, aber in zwei Gaben, mit einem Intervall von 24Stunden verteilt, ohne Vergiftungserscheinungen ertragen werden. Die endliche Wirkung hängt demnach von den Verteilungs- und Ausscheidungsverhältnissen während der Latenzzeit ab, vielleicht davon, wie stark sich die giftempfindlichen Elemente im Herzen während der Latenzzeit der Wirkung mit dem Gifte gleichsam beladen. So erklärt es sich, daß die gleiche Digitoxinmenge von $0,08 \mathrm{mg}$ pro $\mathrm{kg}$, die im oben angeführten Versuche tödlich gewirkt hat, auf zwei Injektionen, an zwei aufeinander folgenden Tagen verteilt, nur Pulsverlangsamung, aber keine Vergiftung hervorrief. Dies zeigt der folgende Versuoh 20.

Versuch 20. 17. Februar 1903.

$2 \mathrm{mal} 0,04 \mathrm{mg}$ pro $\mathrm{kg}$ Digitoxin. Katze $3600 \mathrm{~g}$ Gewicht.

17. Februar 1903225 Pulse. Injektion von $0,04 \mathrm{mg}$ pro $\mathrm{kg}$.

18. $=\quad=180=2$. Injektion von $0,04 \mathrm{mg}$ pro kg.

19. $=\quad=174=$ Keinerlei Nebenwirkungen.

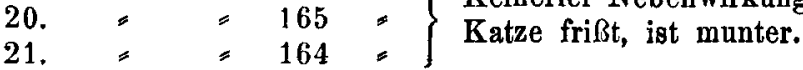


22. Februar 1903.198 Pulse. Gewiebt $3780 \mathrm{~g}$.

24. $=204$.

27. $=204=$ Bleibt gesund.

Die Pulsverlangsamung nach den "therapeutischen" Dosen in diesem Versuch hielt sonach 4 Tage lang an; trotz dauernder Herzwirkung zeigte das Tier dabei keinerlei Vergiftungssymptome und nahm an Gewicht zu.

Auch noch kleinere Gaben haben eine Nachwirkung. Gibt man endlich so kleine Dosen täglich fort, daß jede Gabe als Einzeldosis gereicht, an und fur sich noch unwirksam wäre, so summieren sich diese Nachwirkungen und allmählich tritt die Pulsverlangsamung ein. Es scheint sogar, daß man mit Digitoxin uberhaupt nur dann toxische Nebenwirkungen vermeiden kann, wenn die Pulsverlangsamung durch Summierung mehrerer als Einzeldosen noch nicht wirksamer Gaben zustande kommt. Wenigstens ist es mir bei Anwendung einer einmaligen Digitoxindosis, bei der das Tier im ubrigen gesund geblieben wäre, niemals gelungen, eine deutliche Herzwirkung zu erzielen. Für die Einzeldosis liegt eben infolge der starken Nachwirkung die Dosis toxica zu nahe der eben wirksamen Dosis und so gelingt es leichter durch eine Reihe kleinerer Gaben die gewtinschte Wirkung hervorzurufen als durch eine einmalige Gabe. Injiziert man z. $\mathrm{B}$. $0,02 \mathrm{mg}$ pro $\mathrm{kg}$ täglich wie in dem folgenden Versuche 40 (S. 91), so fübren diese kleinen Gaben erst am 5. Tage zu deutlicher Pulsverlangsamung; ist aber ihre Wirkung einmal durch Kumulierung eingetreten, so dauert sie ebenso wie die Nachwirkung der Einzeldosen fort, wenn nun auch zwei Tage lang die weitere Zufuhr unterbleibt. Eine weitere Anbäufung, welche die tägliche "therapentische" Dosis zu einer toxischen werden ließe, fand in diesem Versuche nicht so bald statt; die Verstärkung und Verlangsamung der Herzschläge konnte vielmelr 3 Wochen lang mit der täglichen Gabe von $0,02 \mathrm{mg}$ festgehalten werden, ohne daf Vergiftungserscheinungen aufgetreten wären.

Versuche wie der auf S. 92 wiedergegebene sind analog der fortgesetzten Darreiohung kleiner Digitalisgaben am Menschen, wie sie von Naunyn ${ }^{1}$ ), Groedel ${ }^{2}$ ), Kussmaul ${ }^{3}$ ) u. a. empfohlen wurde. Der Versuch zeigt, daß man auob von dem sehr stark kumulativ wirkenden Digitoxin eine tägliche Gabe ermitteln kann, durch die

1) Naunyn, Therapie der Gegenwart. 1899.

2) Groedel, Verhandl. d. 17. Kongr. f. inn. Med. Karlsbad 1899.

3) Kußma u l, Therapie der Gegenwart. 1900. 


\section{Versuch 40. 11. Februar 1902.}

Katze $1500 \mathrm{~g}$. $0,02 \mathrm{mg}$ pro kg Digitoxin täglich.

11. Februar 230 Pulse Injekt. a. m.

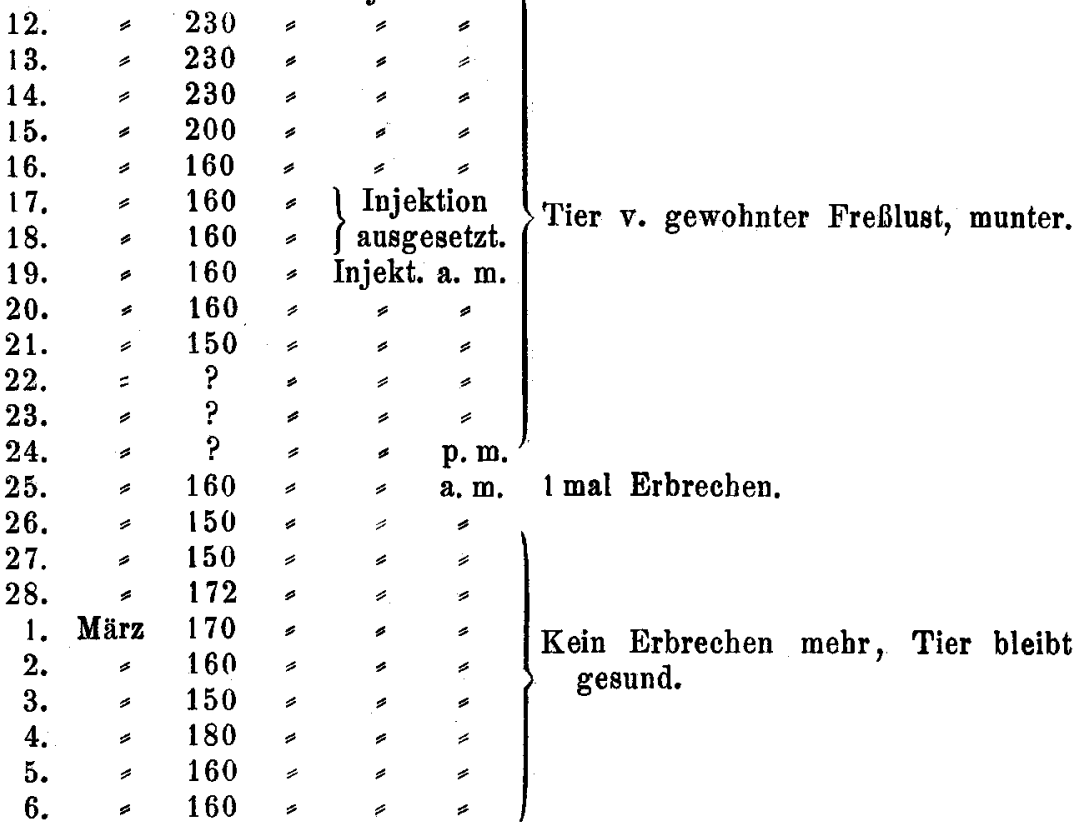

sich einige Wochen hindurch eine dauernde Kreislaufswirkung ohne Nebenerscheinungen erzielen läßt. Doch ist es gerade beim Digitoxin sehr schwer, bei längerer Versuchsdauer eine gefährliche Kumulierung za vermeiden. Wie nahe man z. B. im vorher angefthihten Versuch bei der fortgesetzten Zufuhr von kleinen, erst nach der ftunften Dosis wirksamen Gaben bereits der Gefahr einer toxischen Anhäufung steht, wird u. a. dadurch erwiesen, daß es in diesem Falle genügte, die Zwischenzeit zwischen den zwei Injektionen an zwei aufeinander folgenden Tagen nicht wie sonst, zu 24 Stunden, sondern einmal zu 14 Stunden zu bemessen, um alsbald als erstes Symptom vorlibergehender. Vergiftung Erbrechen auszulösen.

Wählt man die täglichen Gaben ein wenig größer, so machen sich schon nach wenigen Tagen neben der Pulsverlangsamung und Verstärkung der Herzaktion auch toxische Nebenwirkungen geltend. Als Beleg führe ich einen Versuch mit der täglichen Dosis von $0,03 \mathrm{mg}$ pro $\mathrm{kg}$ an, in dem die Pulsverlangsamung entsprechend rascher, schon nach 2 Injektionen, deutlich war, aber im Gegensatz zu dem vorangehenden Versuch mit $0,02 \mathrm{mg}$ schon nach der dritten Injektion 
Erbrechen eintrat und das Tier bei der weiteren täglichen Zufuhrung der Dosis schwer erkrankte.

Versuch 9. 9. Dezember 1902.

1. Tägliche Injektion von $0,03 \mathrm{mg}$ pro $\mathrm{kg}$ Digitoxin.

9. Dezember 225 Pulse. Gewicht der Katze $2450 \mathrm{~g}$.

10. $=210=$

11. $=156=$ Nach der 2. Injekt. am 3. Tage Verlangsamg. u. deutl. verstärkte Herzaktion, im Anschl. an die Injektion dieses Tages tritt Erbrechen ein.

12. $=144=$ Irregulärer Puls.

13. $=156=$ Verminderte Freßlust.

14. $=144=$ Gewicht auf $2100 \mathrm{~g}$ gesumken, frißt nicht mehr.

15. $=168=$

16. $=156=$

18. $=168=$ Katze sehr krank, Versuch abgebrochen.

Die in dem Versuche angewandte Dosis liegt dabei als Einzeldosis noch weit unter der toxischen Grenze. Dennoch tritt die Kumulierung verhältnismäßig rasch ein. Je größer bei täglicher Zufubr die Gaben sind und je rascher sie sich folgen, desto sohneller geht die therapeutische Wirkung in eine toxische tiber. Die Resultate des Tierexperiments stehen somit auch hier in völliger Ubereinstimmung mit den Erfahrungen am Menschen. Die Gesetzmäßigkeit dieser Erscheinungen tritt an dem gleichmäßigen Tiermaterial so deutlich hervor, daß es sich gleichsam quantitativ verfolgen läßt, wie ein Überschreiten der zulässigen Dosis nach oben in wachsender Schnelligkeit zu toxisehen Erscheinungen und zum Tode fuhrt. Das Gesagte wird durch eine Tabelle belegt, in der ich die Wirkungen täglicher Digitoxingaben zusammenstelle.

Tabelle: Wirkung täglicher Digitoxingaben.

\begin{tabular}{|c|c|c|c|c|c|}
\hline Versuch & $\begin{array}{c}\text { Digitoxin } \\
\text { gegeben p. K. }\end{array}$ & $\begin{array}{c}\text { Nach wie- } \\
\text { viel Tagen } \\
\text { Puls- } \\
\text { verlang- } \\
\text { samung? }\end{array}$ & $\begin{array}{c}\text { Nach } \\
\text { wieviel } \\
\text { Gaben Er- } \\
\text { brechen? }\end{array}$ & $\begin{array}{c}\text { Nach } \\
\text { wieviel } \\
\text { Gaben } \\
\text { krank? }\end{array}$ & Bemerkungen \\
\hline $\begin{array}{r}40 \\
9\end{array}$ & $\begin{array}{r}22>00,02 \\
9>00,03\end{array}$ & $\begin{array}{l}5 \\
2\end{array}$ & $\begin{array}{c}\text { nicht } \\
3\end{array}$ & $\begin{array}{c}\text { nicht } \\
9\end{array}$ & \multirow{6}{*}{$\begin{array}{l}\text { bleibt gesund. } \\
\text { abgebrochen, als Tier } \\
\text { schwer krank. } \\
\text { bleibt gesund. } \\
\text { Tod nach } 16 \text { Gaben. } \\
\text { nach } 5 \text { Gaben abge- } \\
\text { brochen, schw. krank. } \\
\text { Tod naoh } 7 \text { Gaben. } \\
\text { Tod nach } 7 \text { Gabcn. }\end{array}$} \\
\hline 20 & $2>0,04$ & 2 & nicht & nieht & \\
\hline 44 & $16 \times 0,04$ & 4 & 6 & 6 & \\
\hline 11 & $5 \times 0,041$ & 3 & 5 & 5 & \\
\hline 8 & $7 \times 0,05$ & $3-4$ & $3-4$ & 4 & \\
\hline 10 & $7 \times 0,087$ & 1 & 2 & 3 & \\
\hline
\end{tabular}


Versuche mit Digitalinum verum.

Auch das Digitalinum verum (Böhringer und Söhne) wurde in alkoholischer Lösung angewandt, die alkoholischen Stammlösungen vor dem Gebrauche mit Wasser verdunnt.

Das Digitalin scheint rascher als Digitoxin zu den giftempfindlichen Apparaten im Herzen vorzudringen. Nach einer einmaligen, etwa minimal-letalen Dosis von $0,48 \mathrm{mg}$ pro $\mathrm{kg}$ ist zwar die Pulsverlangsamung in den ersten 24 Stunden noch nicht nachweisbar, sie wird aber jedenfalls nach weniger als 48 Stunden manifest. Ist die Wirkung auf den Puls einmal erreicht, so wird sie nach Digitalin ebenso lange festgehalten, als nach Digitoxin. Bèides wird durch nebenstehenden Versuch 15 gut illustriert.

Das Diagramm zeigt, wie sich die Pulsverlangsamung nach ihrem Eintritt noch 3 Tage lang vertieft bis auf die Hälfte der Normalfrequenz; allmählich nimmt die Pulszahl dann wieder zu, bleibt aber noch zwei Tage unter der Norm, während das Tier krank wird und endlich unter Pulsbeschleunigung zugrunde geht. In bezug auf die Nachwirkung ungefähr physiologisch gleichwertiger Gaben besteht also jedenfalls kein wesentlicher Unterschied zwischen beiden Präparaten. Auch sonst gilt von den kumulativen Eigenschaften des Digitalin im wesentlichen das beim Digitoxin Gesagte. Auch hier werden kleine Dosen, die als Einzelgaben gereicht noch unwirksam wären, bei der fortgesetzten Darreichung wirksam und wirksame Gaben werden bei täglicher Zufuhr toxisch. Doch zeigt das Digitalin bei täglicher Einverleibung insofern einen Unterschied, als es in solchen Reihenversuchen mit Digitalinum verum weit leichter als mit Digitoxin gelingt das therapeutisch verwertbare stadium der Herzwirkung festzuhalten, ohne daß die Tiere erbrechen oder krank werden. Ich verfüge tiber zwei derartige Versuche von langer Dauer. In dem einen (Versuch 42) erhielt eine Katze innerhalb 25 Tagen 23 Gaben von 0,11 mg pro kg Digital. verum; nach der neunten Gabe trat durch Kumulierung Pulsverlangsamung ein, allmählich ging die Pulszahl bis 100 herab und diese Verlangsamung und Verstärkung der Herzaktion konnte noch wochenlang festgehalten werden, ohne daß Nebenersoheinungen auftraten. Noch eklatanter ist der zweite Fall, in dem das Versuchstier vom 1. November 1902 bis zum 31. Januar 1903, also 92 Tage lang, täglich $0,2 \mathrm{mg}$ pro $\mathrm{kg}$ seines Anfangsgewichts erhielt und trotz dauernder und beträchtlicher Pulsverlangsamung von anderweitigen Vergiftungssymptomen frei blieb. Während der ganzen Zeit trat niemals Erbrechen auf, die Freßlust war nicht gestört, das Körpergewicht der 
Versuch 15. 26. Januar. 1903. Wirkung einer einmaligen Dosis Digitalinum verum.

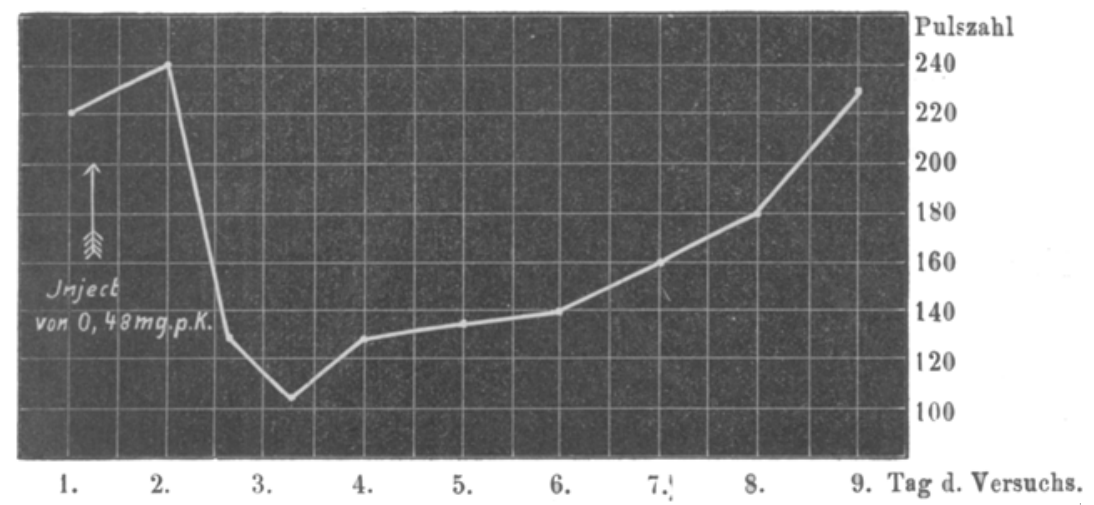

Fig. 3.

Kardiographische Kurven zu Versuch.15.

Normal $=216$ Pulse pro Min.

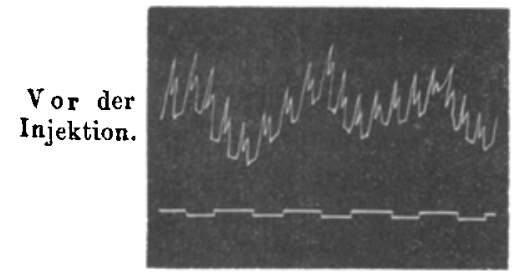

130 Pulse pro Min.

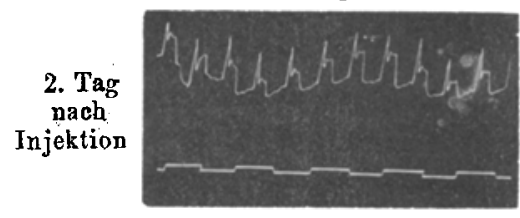

138 Pulse pro Min:

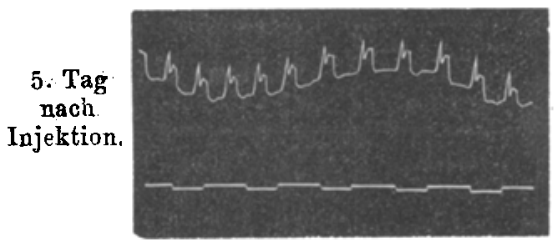

2. Tag (p. m.)

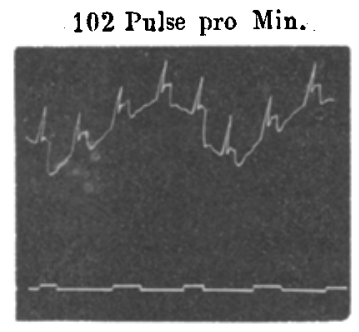

228 Pulse pro Min.

8. Tag nach Injektion.

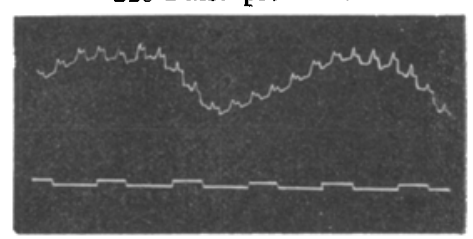

Fig. 4. 
Katze nahm sogar zu. Dennoch war man auch in diesem Versuche von der Gefahr toxischer Anhäufung nicht sehr weit entfernt. Denn als nach der Zeit von 92 Tagen die tăgliche Dosis nur um $1 / 5$ gesteigert wurde, änderte sich alsbald das Bild; vortibergehend nahm die Pulsfrequenz noch weiter ab, aber am siebenten Tage nach der Erhöhung der Gabe trat zum erstenmal als Symptom der eingetretenen Kumulation Erbrechen auf. Weiterhin stieg die Pulsfrequenz plötzlich an und ging sogar tiber die Norm, das Tier wurde krank, nahm bedeutend an Gewicht ab, erholte sich aber, als nun die Injektionen ausgesetzt wurden, wieder vollstăndig unter erneutemHerabgehen derPulsfrequenz. Ein Auszug aus dem Protokoll mag den Versuch näher belegen; die Pulszahlen sind jedesmal durch Auszählen des Kardiogramms ermittelt.

Versuch 2. Tägliche Gaben von $0,2 \mathrm{mg}$ Digital. ver. pro kg.

\begin{tabular}{|c|c|c|c|c|}
\hline Datum & Pulszabl & Gew. in $\mathrm{g}$ & Injektion & Bemerkungen \\
\hline 1. XI. 02. & 204 & 2000 & täglich & \\
\hline 14. XI. & 132 & - & - & \\
\hline 2. XII. & 168 & - & 一 & bei konstanter Pulswirkung \\
\hline 7. XII. & - & 2400 & - & Tier gesund. \\
\hline 31. XII. & 168 & 2450 & - & \\
\hline $\begin{array}{l}29.1 .03 . \\
2.11 .\end{array}$ & $\begin{array}{l}156 \\
165\end{array}$ & $\overline{2400}$ & - & da das Tier um $1 / 5$ seines Körperge- \\
\hline & & & & $\begin{array}{l}\text { wichtes zugenommen hat, wird die } \\
\text { täglicho Dosis erhöht, d. h. in das Ver- } \\
\text { hältnis zum Körpergewicht gebracht. }\end{array}$ \\
\hline 3. 11. & 156 & - & - & \\
\hline 4. 11 . & 126 & - & - & \\
\hline 5. II. & 180 & 一 & - & \\
\hline $\begin{array}{l}7.11 . \\
14.11 .\end{array}$ & 174 & $\overline{1940}$ & keine & $\begin{array}{l}\text { Erbreohen } \\
\text { T. krank, daher Injekt, a usgesetzt. }\end{array}$ \\
\hline 18. III. & - & 2200 & 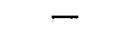 & Tier wieder gesund. \\
\hline
\end{tabular}

Dieser Versuch, in dem auch nach einer 92 tägigen Digitalinbehandlung die geringftigige Steigerung der Dosis um $1 / 5$ alsbald zu Vergiftungserscheinungen führte, spricht sehr gegen die von v.d.Heid $\mathrm{e}^{\text {1) }}$ in weit kürzeren Versuchsreihen behauptete Gewöhnung an das Gift. Die kumulativen Eigenschaften des Digitalins bei täglicher Zufuhr etwas größerer Gaben werden durch die folgende Tabelle illustriert.

Tabelle: Wirkung täglicher Digitalingaben.

\begin{tabular}{|c|c|c|c|c|}
\hline Versuch & $\begin{array}{l}\text { mg Digitalin } \\
\text { gegeben pro kg }\end{array}$ & $\begin{array}{l}\text { Nach wieviel } \\
\text { TagenPulsver- } \\
\text { langsamung? }\end{array}$ & $\begin{array}{c}\text { Nuch wieviel } \\
\text { Tagen Er- } \\
\text { bronben? }\end{array}$ & Weiterer Verlauf \\
\hline $\begin{array}{r}42 \\
2 \\
49\end{array}$ & $\begin{array}{l}23 \times 0,11 \\
92 \times 0,2 \\
20<0,21 \\
7 \times 0,4\end{array}$ & $\begin{array}{l}9 \\
? \\
5\end{array}$ & $\begin{array}{c}\text { nicht } \\
\text { nicht } \\
13 \\
2\end{array}$ & $\begin{array}{l}\text { bleibt gesund. } \\
\text { bleibt gesund. } \\
\text { ron d. 15. Gabe an sehwer } \\
\text { krank, abgebrochen. } \\
\text { Tod nach } 22 \text { 'Tagen. }\end{array}$ \\
\hline
\end{tabular}

1) A. a. 0 . 


\section{Versuche mit Strophanthin.}

Es kamen meist Strophanthin Merek und Strophanthin Böhringer in wässriger Lösung zur Anwendung. Zwischen beiden Präparaten war weder in der Wirkungsstärke noch im ubrigen Verhalten ein Unterschied zu konstatieren.

Das leichtlösliche Strophanthin weicht in bezug auf den Verlauf der Kreislanfsveränderungen in einem Punkte von Digitalin nnd noch mebr von Digitoxin ab: Die Verlangsamung und Verstärkung der Herztätigkeit setzt nach einer in der Wirksamkeit vergleichbaren Strophantbindosis sebr viel rascher ein als nach Injektion der anderen Substanzen. Nach Einzelgaben von Strophanthin, die noch nicht toxisch wirken, tritt die Pulsverlangsamung schon nach 4-5 Stunden ein, nach toxischen Gaben natürlich noch rascher. Um mit Digitalin oder Digitoxin schon nach Stunden die Kreislaufswirkung zu erzwingen, müssen wir die Versuchstiere mit mehrfach letalen Dosen tiberschwemmen; nur wenn solche mit der Fortdauer des Lcbens unverträgliche Giftmengen zirkulieren, tritt die Pulswirkung bei diesen substanzen schon nach Stunden auf. Bei der minimal-letalen Dosis Digitalimum verum hingegen dauert es uber 24 Stunden, bei Digitoxin noch weit länger, bis die Wirkung auf den Puls manifest wird. Das Strophanthin erreieht also weit sehneller die giftempfindlichen Apparate im Herzen oder tritt mit ihnen weit rascher in Reaktion.

Auch an dem zeitlichen Auftreten, des Erbrechens, des regelmäBigen Initialsynıptoms der Vergiftung, war es deutlich erkennbar, daß Strophanthin ungleich rascher zur Wirkung kommt als Dizitoxin. In einer größeren Anzahl von Einzelversuchen wurde die Zeit notiert, die zwischen der Injektion etwa eben toxischer Gaben beider Substanzen und dem Eintritt des Erbrechens verstrich. Wäbrend dasselbe nach Digitoxin nie vor einer Stunde erfolgte, konnte es nach Strophanthin schon 10-20 Minuten nach der Injektion beobacbtet werden. Das Strophanthin ist aber nur durch den rascheren Eintritt der PuIsveränderung ausgezeichnet. Ist die Kreislaufswirkung einmal eingetreten, so wird sie auch nach Strophanthin tagelang festgehalten. Auch dem Strophanthin kommt also eine ausgeprägrte Nachwirkung zu. Einige Versuchsprotokolle mögen den raschen Eintritt und die lange Dauer der Wirkung bei kleinen, noch nicht toxischen, sowie-nach vergiftenden Dosen illustrieren. 
Versuch 21. 23. Febr. 1903. Wirkung einmaliger Dosis Strophanthin Merck 0,05 pro kg.

Normal $=229$ Pulse pro Min.

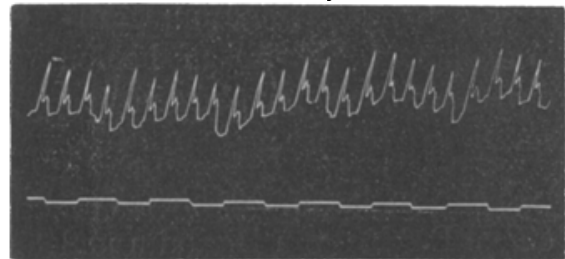

4 Stdn. nach Injekt $=184$ Pulse pro Min.

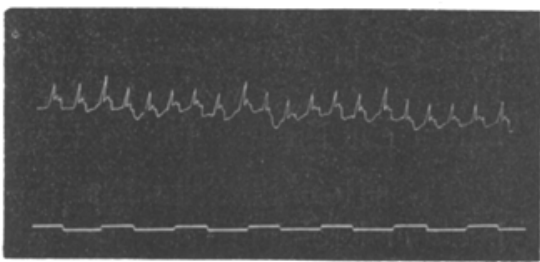

Fig. 5.

Versuch 26. 12. März 1903. Wirkung einmaliger Dosis Strophanthin Merck, 0,08 pro $\mathrm{kg}$.

12. März. Normal $=228$ Pulse pro Min.

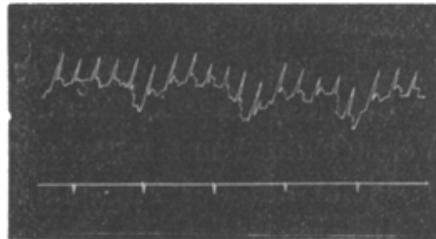

30 Stdn. nach lnjekt. 168 Pulse pro Min.

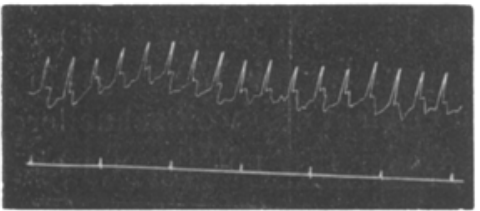

Versuch 61. 24. März 1902 thin Thoms, 0,2 $\mathrm{mg}$ pro $\mathrm{kg}$. 24. Marz. Normal $=205$ Pulse pro Min.

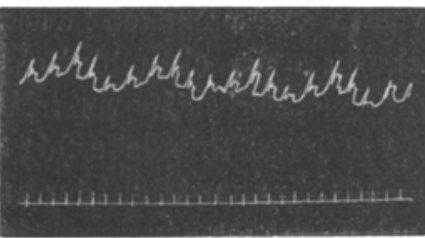

25. Mürz. 24itdn. nach Inj. 147 Pulse pro Min.

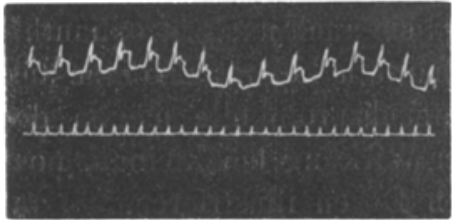

13. März. 24 Std. nach Inj. 186 Pulse pro Min.

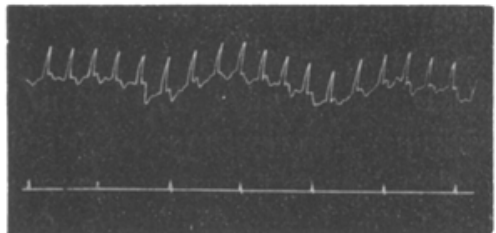

14. März. 2 Tage nach Injekt. 216 1"ulse pro Min.

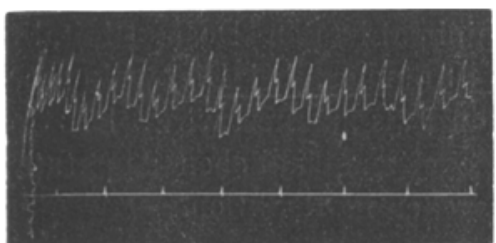

Wirkung einmaliger Dosis Strophan-

4 Stdn. nach Injekt. 135 Pulse pro Min.

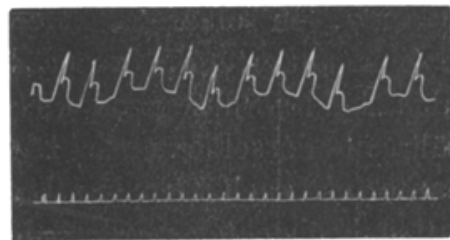

26. Măr. 48 Stdn. nacb Inj. 151 l’ulse pro Min.

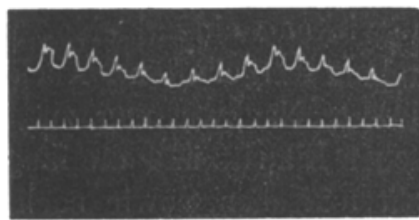

Fig. 6 . 
Das Bestehen einer langen Nachwirkung beweist zugleich, daß auch dem Strophanthin kumulative Eigenschaften zukommen müssen; denn bei fortgesetzter Anwendung eben wirksamer Gaben mussen sich deren Nachwirkungen summieren. In der Tat tritt nach Strophanthin ebenso wie nach den anderen Substanzen der Gruppe Kumulation ein; an und fur sich ungiftige Einzelgaben werden bei täglicher Zuftuhrung toxisch. Die folgenden Versuche sind dafür ein Beleg.

\section{Versuch 5. 26. November 1903.}

Tägliche Gaben von 0,03 pro kg Strophanthin Merck. 26. November Injekt.
27. $=$
28. $=$ do.
29. $=$
do. Tier gesund bei exquisiter Pulsverlangsamung.
30. = keine
1. Dezember Injekt.
2. = do. Einige Stunden nach Injekt. Erbrechen.
3. $=$ do. Sofort nach Injekt. Erbrechen, Tier krank.

\section{Versuch 3. 14. November 1903.}

Tägliche Gaben von 0,05 pro kg Strophanthin Merck. 14. November Injekt.
15. $=$
do.
16. $=$
do.
Tier gesund bei erheblicher Pulsverlangsamung.
17. $=$
18. $=$
do.
do.
19. $=$
do. Erbrechen nach Injekt., Tier krank.

Das Tierexperiment bestätigt somit die ärztliche Erfahrung, daß die Strophanthinwirkung rasch eintritt. Dagegen trifft die weitverbreitete Annahme, daß das Mittel nicht kumulativ wirke, sicherlich nicht zu. Zum Teil ist diese Vorstellung; daß bei Strophanthin die Gefahr der Kumulation weniger drobe, wobl dadurch entstanden, dab vielfach und insbesondere in Dentschland sehr unwirksame Tinkturen in Gebrauch sind ${ }^{1}$ ). Bei stark wirksamen Präparaten wird aber die rasch eintretende Wirkung ein frühzeitiges Warnungssignal sein; das von weiterer Zufuhrung abhält.

\section{Zusammenfassung.}

Die vorliegenden Untersuchungen verfolgen die chronischen Wirkungen der wichtigsten reinen Digitaliskörper an einem gleichmäßigen Tiermaterial und in untereinander gut vergleichbaren

1) Vergl. Fraenkel, Therapie der Gegenwart März 1902. 
Versuchsreihen. Apbaltspunkte zur Annahme einer Angewöhnung wurden dabei nicht gewonnen, bingegen treten die kumulativen Wirkungen deutlich hervor. Dabei mub vor allem hervorgeboben werden, mit welcher Gesetzmäßigkeit sich die Erscheinungen der Kumulation entwickeln, d.h. wie mit ansteigender Gabe und in Abbängigkeit von dem Abstand der Gaben unter einander die Vergiftungserscheinungen sich immer rascher einstellen. Im Vergleich zu anderen toxikologischen Gruppen spielt bei den Digitaliskörpern die Individualität des einzelnen Versuchstiers eine sehr geringe Rolle.

Prinzipiell verhalten sich die untersuchten Substanzen in ihren kumulativen Eigensehaften gleich. Ftir jeden der untersuchten Digitaliskörper lïßt sich eine bestimmte tägliche Gabe ermitteln, durch die auch bei wochenlanger Einfuhrung das "therapentische Stadium" festgebalten wird; die Übersclreitung dieser Tagesdosen um ein geringes führt aber auch bei allen Substanzen zu toxischer Kumulierung. Dennoch ist die Neigung zur Kumulation bei den einzelnen Körpern graduell verschieden. Man kann zu einer Vorstellung tiber den Grad der kumulierenden Wirkung gelangen, wenn man die Größe der wirksamen E inzelgabe mit jener Dosis vergleicht, die man einige Zeit lang täglich fortgeben kaun, ohne Vergiftungserscheinungen zu erzeugen. Für Digitoxin einerseits und Digitalin andererseits ergibt sich dieser Vergleieh aus den oben angeführten Versuchen. Wie die Versuche 18 und 15 zeigen, sind $0,08 \mathrm{mg}$ Digitoxin pro $\mathrm{kg}$ und $0,48 \mathrm{mg}$ Digitalinum verum als physiologisch etwa gleiehwertige und zwar als einfach-letale Einzelgaben beider Körper zu bezeichnen. Bei Digitoxin wirkt aber schon weniger als $1 / 3$ dieser Einzelgabe nach der 3. Injektion toxisch (Versuch 9 mit $0,03 \mathrm{mg}$ pro Kilogramm) und macht das Tier nach 9 Tagen schwer krank, wäbrend man von Digitalin fast die Hälfte der Einzelgabe (Versuch 2 mit $0,2 \mathrm{mg}$ pro $\mathrm{kg}$ ) wochenlang fortgeben kann, ohne daß Vergiftungserscheinungen auftreten. Der Abstand täglich gereiehter wirksamer Gaben von der wirksamen Einzelgabe muß sonach bei Digitoxin beträchtlich größer sein als bei Digitalin, wenn man Vergiftungserscheinungen vermeiden will; Digitoxin kumuliert also stärker. Auch für die Einzeldosis ist beim Digitoxin der Abstand zwischen wirksamer und tödlicher Gabe ein so geringer, daß es mir nicht gelang durch die nachfolgende Anwendung einer einzigen Gabe Pulsverlangsamung ohne tödliche Vergiftung zu erzielen. Bei Digitalin und bei Strophanthin gelingt dies lingegen leicht. 
Alle Digitalinkörper scheinen die Eigenschaft der kumulativen Wirkung zu besitzen. Wenigstens kommt sie auch dem so leicht wasserlöslichen Strophanthin nach meinen Versuchen, dem Helleborein nach denen v. d. Heides zu. Diese Tatsache wirft auch einiges Licht auf die Ursachen der Kumulation. Man hat den Grund für die sukzessive Vertiefung der Wirkung bei fortdauernder Zufuhr kleiner Dosen in einer Anhäufung des Giftes im Blute gesucht; eine derartige Anbäufung könnte durch eine im Verhältnis zur Aufnahñe verzögerte Ausscheidung bedingt sein. Dann wäre zu erwarten, daß besonders die schwer löslichen Substanzen wie sie im Vergleich zu leicht löslichen einen verzögerten Eintritt der Wirkung zeigen, auch die Kumulation weit stärker hervortreten lassen. Vergleichen wir von diesem Gesichtspunkte aus physiologisch gleichwertige, d. b. in ihrer Wirkung auf das Herz ungefähr gleichstarke Gaben von Digitoxin, Digitalinum verum und Strophanthin, so ergibt sich, daß die Schnelligkeit, mit der die Wirkung eintritt, in der Tat mit der Löslichkeit parallel geht. Anders aber steht es mit der Dauer der einmal eingetretenen Wirkung und mit dem kumulativen Charakter der Substanzen. In dieser Beziehung verwischen sich die Unterschiede, wie ein Blick auf die starke Nachwirkung und auf die kumulierende Wirkung in unseren Strophanthinversuchen zeigt. Man wird dadurch zu der Annahme gedrängt, daß die kumulative Wirkung nicht so sehr von dem Grade der Ausscheidbarkeit aus dem Blute als vielmehr von der Festigkeit der einmal im giftempfindlichen Gewebe des Herzens entstandenen Bindung abhängt. Zu ähnlichen Vorstellungen gelangten tubrigens auch Stokvis und v. d. Heide durch Feststellung der kumulativen Eigenschaften des leicht löslichen Helleboreins.

Von der Schnelligkeit, mit der das Herz die einzelnen Digitaliskörper aufnimmt, hängt es ab, wie lange es dauert, bis sie wirken, von der Festigkeit der Bindung im Herzen aber, wie lange die einmal eingetretene Wirkung anhält. Die Veränderungen der Herztätigkeit dauern in allen Fällen lange an; der Eintritt hingegen erfolgt bei den verschiedenen Präparaten verschieden rasch. Nach Strophanthin läßt sich die Kreislaufswirkung nach einer "therapeutischen" Dosis schon nach wenigen Stunden nachweisen, nach Digitalin in gleichwertiger Gabe dauert es tiber 24 Stunden und nach Digitoxin noch weit länger, bis tuber 60 Stunden. Diesem Momente kommt auch praktische Bedeutung zu. Wo man rasche Wirkung erzielen will, scheint Strophanthin am geeignetsten. Auch eine gefahrvolle Überdosierung bei fortgesetzter Anwendung wird sich im allgemeinen 
leichter vermeiden lassen, wenn sich eine geringe Überschreitung der therapeutischen Grenzen schon nach wenigen Stunden durch toxische Erscheinungen kundgibt. In diesem Sinne erscheinen Strophanthin und Digitalin zu dauernder "chronischer" Digitalistherapie eher geeignet als Digitoxin.

Will man also aus den vorliegenden Untersuchungen Sohlüsse fur die therapeutisehe Anwendbarkeit der reinen Digitaliskörper ziehen, so ergibt sich, daß Digitoxin in wenigen, vorsichtig dosierten Gaben fur eine kurzdauernde energisohe Kur geeignet erscheint, nicht aber für länger dauernde Behandlung. Digitalin und wohl auch Strophanthin eignen sich hingegen in richtig gewählter Dosis auch zu täglicher Darreichung durch längere Zeit. Strophanthin empfiehlt sich dort, wo man die Kreislaufswirkung rasch erzielen will; aber auch bei Strophanthin ist die Gefahr der Kumulation vorhanden.

Es ist zu boffen, daß solche experimentelle und ahnliche klinische Studien dazu beitragen werden, die aus vielen Grtunden erstrebenswerte Anwendung reiner Digitalissubstanzen zu fördern. 